\title{
Development trends of combined inductance-capacitance electromechanical energy converters
}

\author{
Hamlet Karayan and Seyran Gandilyan* \\ Department of Optics, Yerevan State University, Armenia
}

\begin{abstract}
In the article the modern state of completely new direction of electromechanical science such as combined inductive-capacitive electromechanics is considered. The wide spectra of its possible practical applications and prospects for further development are analyzed. A new approach for mathematical description of transients in dualcon jugate dynamic systems is proposed. On the basis of the algorithm differential equations for inductive-capacitive compatible electromechanical energy converters are derived. The generalized Lagrangian theory of combined inductively-capacitive electric machines was developed as a union of generalized Lagrangian models of inductive and capacitive electro-mechanical energy converters developed on the basis of the basic principles of binary-conjugate electrophysics. The author gives equations of electrodynamics and electromechanics of combined inductive-capacitive electric machines in case there are active electrotechnical materials of dual purpose (ferroelectromagnets) in the structure of their excitation system. At the same time, the necessary Lagrangian for combined inductive-capacitive forces was built using new technologies of interaction between inductive and capacitive subsystems. The joint solution of these equations completely determines the dynamic behavior and energy characteristics of the generalized model of combined machines of any design and in any modes of interaction of their functional elements
\end{abstract}

\section{Introduction}

Back in 1980-90-ies in the writings of Academician A.G. Iosifyan and Professor I.P. Kopylov on the basis of a critical analysis of the physical and technical foundations of the processes of generation, transmission, conversion and consumption of electromagnetic energy in inductive (magnetically induction) and capacitive (electro-induction) electric machines and apparatuses the possibility of implementing a third type of electromechanical energy converter has been installed - combined inductivecapacitive (magneto-electric induction), in which fluxchains (magnetic and electric) play an important role during the energy-exchange processes $[1,2]$.

The development of modern electromechanical science at the junction of the latest achievements of classical mechanics, electrodynamics, solid state physics, electrotechnical materials science, semiconductor physics, atomic physics, chemistry, cybernetics, etc., shows that the possible practical embodiment of the idea of inductive capacitive electromechanics currently predominates and can provide a new qualitative leap in the development of those branches of human activity, the basic value for which has a complex application ix systems electromechanical energy converters (from medical robotics to create a means of space exploration) [3-5].
Practical implementation of the idea of inductive capacitive electromechanics can be strongly influenced by the development of the main direction of nanoscience - nanostructural material science (nanocrystalline, nanocomposite, nanofibrous, etc.) [6,7].

System analysis of fundamental research conducted at the turn of the 20th and 21st centuries in the field of physico-mathematical modeling, automated design and manufacturing of inductive-capacitive components of a microsystem electric drive is given by some foreign and Russian scientific publications, for example in [8-14].

In this paper, which is an organic continuation of the work of the authors [14,15], a new approach to the solution of some nodal questions of the generalized physico-mathematical modeling of combined inductive-capacitive energy converters allowing to take into account a large number of interrelated factors that determine their dynamic and energy characteristics is proposed.

\section{Equations of electromechanical connections in combined inductive capacitive electromechanical energy converters}

In [16-18], the generalized Lagrangian theory of generalized inductively-capacitive electric machines was developed as a union of generalized Lagrangian

\footnotetext{
Corresponding author: angastroi@mail.ru
} 
models of inductive and capacitive electromechanical energy converters, developed on the basis of the basic principles of binary-conjugate electrophysics.

Let us consider a more correct formulation of this problem. Without loss of generality, we can assume that the generalized combined machine, which is under consideration, consists of four contour chains: two stator symmetric three-phase circuits $(\hat{S}-$ inductive, $\hat{R}$ - capacitive phase, denoted by $\hat{a}, \hat{b}, \hat{c}$ and $\widehat{m}, \widehat{n}, \hat{h})$, respectively, two rotor excitation circuits of a constant magnetic $-\hat{F}$ and electric $\hat{P}$ field (spatially located between systems $\hat{S}$ and $R$ ) (Figure 1).

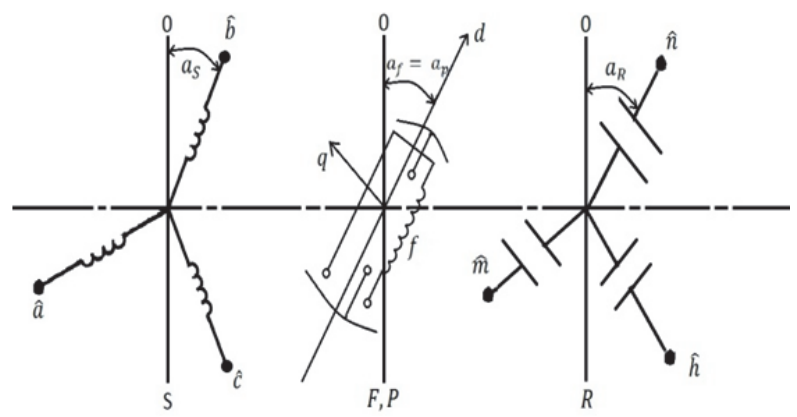

Fig. 1. Scheme of contour circuits of a combined inductivecapacitive machine with a dual excitation system.

Excitation of a constant magnetic flux $\Psi_{f}=\int B d s$ of the inductive part is carried out with the help of an excitation winding $f$, DC powered, the excitation of a constant electric flow $Q_{p}^{*}=\int D d s$ of the capacitive part is carried out by means of dipole electrodes, the power of which is carried out through special contact rings from a constant-voltage source. Between the phase circuits of the two subsystems there is a series (or parallel) galvanic coupling: respectively between $\hat{a}$ and $\hat{m}$ through the resistance $R_{a m} ; \hat{b}$ and $\hat{n}$ through the resistance $R_{b n} ; \hat{c}$ and $\hat{h}$ through the resistance $R_{c h}$ (Figure 2).

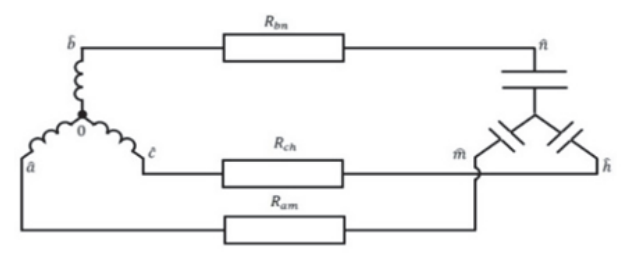

a)
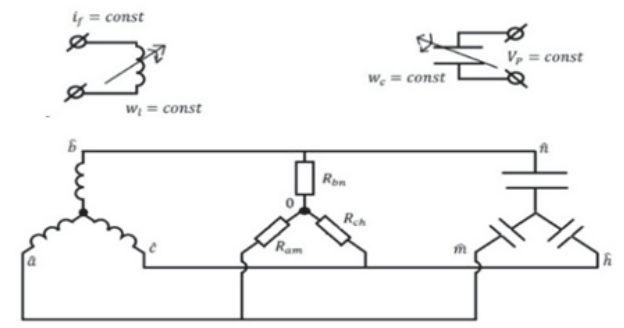

b)
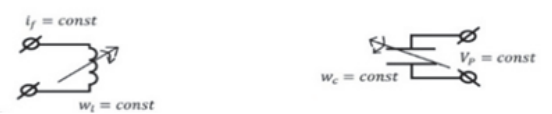

Fig. 2. The electrical circuit of the combined inductivecapacitive alternator: $\mathrm{a}$ - for series connection of phase circuits; $b$ - for parallel connection of phase circuits.
The energy state of a combined machine in the multidimensional phase space of generalized variables is described by means of generalized coordinates - $q$ $\left(\mathrm{q}_{\mathrm{a}}, \mathrm{q}_{\mathrm{b}}, \mathrm{q}_{\mathrm{c}}, \mathrm{q}_{\mathrm{f}}\right.$ ) (as an electric charge in the current cords of an inductive subsystem), $\psi^{*}\left(\psi_{m}^{*}, \psi_{n}^{*}, \psi_{h}^{*}, \psi_{p}^{*}\right)$ (as a magnetic flux "charge" inductive working electric field of a capacitive subsystem) corresponding generalized pulses $\Psi^{*}\left(\Psi_{a}^{*}, \Psi_{b}^{*}, \Psi_{c}^{*}, \Psi_{f}^{*}\right)$ (working magnetic flux), $\mathrm{Q}^{*}\left(\mathrm{Q}_{m}^{*}, \mathrm{Q}_{n}^{*}, \mathrm{Q}_{h}^{*}, \mathrm{Q}_{p}^{*}\right)$ ( working electric flux). As a mechanical generalized coordinate, we take the rotor rotation angle $\gamma=\int_{0}^{t} \omega d t$ or linear deviation $\mathrm{x}=\int_{0}^{t} V d t$.

The output of the equations of the generalized model of a combined machine is based on the Lagrange equations of the second kind describing the behavior of a nonconservative system with lumped parameters:

$$
\begin{gathered}
\frac{d}{d t}\left(\frac{\partial \mathcal{L}}{\partial \dot{a}_{i}}\right)-\left(\frac{\partial \mathcal{L}}{\partial a_{i}}+\frac{\partial D_{0}}{\partial \dot{a}_{i}}\right)=B_{i} \\
i=a, b, c, f, m, n, h, p
\end{gathered}
$$

where $a\left(a_{i}\right)=a\left(\mathrm{q}_{\mathrm{a}}, \mathrm{q}_{\mathrm{b}}, \mathrm{q}_{\mathrm{c}}, \mathrm{q}_{\mathrm{f}} ; \psi_{m}^{*}, \psi_{n}^{*}, \psi_{h}^{*}, \psi_{p}^{*}\right)-$ combined symbolic vector of generalized coordinates; $\dot{a}\left(\dot{a}_{i}\right)=\dot{a}\left(i_{j}=\frac{d q_{i}}{d t} ; V_{j}^{*}=\frac{d \psi_{j}^{*}}{d t}\right), \quad(i=a, b, c, f ; j=$ $m, n, h, p)$ - generalized velocity vector; $\beta\left(\beta_{i}\right)=$ $\beta\left(e_{1}=-\frac{d \Psi_{i}}{d t}, i_{i}^{*}=-\frac{d Q_{i}}{d t}\right)$ generalized forces; $\mathcal{L}-$ Lagrangian energy function; $\mathrm{D}_{0}=-\frac{1}{2}\left(\sum_{j, i}\left(R_{i} i_{i}^{2}+\right.\right.$ $\left.\left.G_{j} V_{j}^{* 2}\right)\right)-$ loss or dispersion function.

From the point of view of energy conversion processes, in principle the following cases can be realized.

1. The mechanical energy is supplied to the system $\widehat{F P}$, the system $\hat{F}$ is excited. Then the combined machine operates in the inductive generator mode.

2. Mechanical energy is supplied to the system $\widehat{F P}$, the system $\hat{P}$ is excited. Then the combined machine operates in the mode of a capacitive generator. 3. Mechanical energy is supplied to the system $\widehat{F P}$, the system $\widehat{F P}$ is excited by a constant current.

Then the system $\widehat{F S}$ operates in the inductive generator mode, $\widehat{P R}$ - in the mode of the capacitive generator.

4. Electromagnetic energy brings systems $\hat{S}$, the system $\hat{F}$ excited by a direct current, the system $\hat{P}-$ by a constant voltage. Then, the system $\widehat{F S}$, operates in the inductive motor mode, and the system $\widehat{P R}-$ in the mode of the capacitive generator.

If in the structure of a combined inductive capacitive electric machine there are no active working materials (such as ferroelectrics and magnets), then it is represented as a simple linear sum of two machines inductive and capacitive, operating on the same shaft and interconnected by anchor electric circuits.

With a linear connection between the inductive and capacitive subsystems, the Lagrange function $\mathcal{L}$ is an additive function.

$$
\mathcal{L}=\mathcal{L}_{L}(x, p, \Psi, q)+\mathcal{L}_{c}\left(x, p, Q^{*}, \Psi^{*}\right) .
$$


In this case, the equations of electrodynamics of a combined machine can be expressed in the following vector-matrix form:

$$
\left.\begin{array}{r}
\frac{d}{d t}\left(\begin{array}{c}
\psi^{*} \\
Q^{*}
\end{array}\right)+\left(\begin{array}{cc}
R_{L} & 0 \\
0 & G_{c}
\end{array}\right) *\left(\begin{array}{c}
i_{L} \\
V_{c}^{*}
\end{array}\right)=\left(\begin{array}{c}
V_{L} \\
i_{c}^{*}
\end{array}\right) \\
\left|\begin{array}{l}
\psi^{*} \\
Q^{*}
\end{array}\right|=\left|\begin{array}{cc}
L & 0 \\
0 & C
\end{array}\right| *\left|\begin{array}{c}
i_{L} \\
V_{c}^{*}
\end{array}\right|
\end{array}\right\}
$$

where $\psi, R_{L}, L$ - submatrices of magnetic flux linkages, active resistances and inductances of a magnetic machine, $Q^{*}, G_{c}, C$ - submatrices of electric flux linkages, active conductivities and capacitances of electrical; $i_{L}, V_{L}$ - vectors of currents and voltages of magnetic circuits, $V_{c}^{*}, i_{c}^{*}$ - vector of voltages and currents of electrical circuits.

The equation of dynamics is supplemented by the equation of motion of the rotor

$$
\begin{gathered}
M=\left(J_{m}+J_{e}\right) \frac{d \omega}{d t}+\frac{K \omega}{P_{0}}+\frac{P_{0}}{2}\left(i_{L}^{\tau} \frac{d L}{d t} i_{L}+\right. \\
\left.V_{c}^{* \tau} \frac{d C}{d t} V^{*}\right) \frac{1}{\omega},
\end{gathered}
$$

where $J_{m}, J_{e}$ - moments of inertia of the rotors of inductive and capacitive subsystems, $P_{0}-$ number of pole pairs.

When the phase start circuits are connected in series, the inductive subsystem as a voltage source is a "carrier system" with an active-capacitive load, and with a parallel connection, the "carrier" system is a capacitive subsystem as a current source with an active-inductive load.

In this case, the equations of electrodynamics of a combined machine can be written in the following form: equations for stator system $\widehat{S R}$ for the serial coupling of phase stator circuits

$$
V_{S P}=\frac{d \Psi}{d t}+R_{L} i_{L}+C_{0}^{-1} \int i_{c} d t,
$$

where the vector $i_{c}^{*}$ - is determined from the systems of equation

$$
\frac{d Q^{*}}{d t}+G_{c} V_{c}^{*}=i_{c}^{*},
$$

matrix $C_{0}-$ is expressed in the form of

$$
\mathrm{C}_{\mathbf{0}}=\left|\begin{array}{ccc}
C_{m m} & 0 & 0 \\
0 & C_{n n} & 0 \\
0 & 0 & C_{h h}
\end{array}\right|
$$

equations for stator system $\widehat{S R}$, with a parallel connection of phase stain chains

$$
i_{R S}^{*}=\frac{d Q^{*}}{d t}+G_{c} V_{c}^{*}+L_{0}^{-1} \int V_{L} d t
$$

where the vector $V_{L}$ - is determined from the system of equation

$$
\frac{d \Psi}{d t}+R_{L} i_{L}=V_{L}
$$

and matrix $L_{0}$ is expressed in the form of

$$
L_{0}=\left|\begin{array}{ccc}
L_{a a} & 0 & 0 \\
0 & L_{b b} & 0 \\
0 & 0 & L_{c c}
\end{array}\right|
$$

\section{Resonance in an inductive capacitive machine}

Resonance in the electrical circuit can be obtained both in parallel connection of the inductive part with the capacitive part - the resonance of the currents, and in their subsequent connection - the resonance of the voltages [19].

In a combined machine that does not contain external power sources, the reactive power is determined by the partial alternating current $-\omega=$ $\omega_{L}=\omega_{C}$ and the maximum values of the energy accumulated in the inductive and capacitive subsystems of the machine:

$$
\left.\begin{array}{c}
P_{L}=V_{L} i_{L} \sin \frac{\pi}{2}=\frac{L i_{L}^{2}}{2}=\omega W_{L(\max )} \\
P_{C}=-V_{C}^{*} i_{C}^{*} \sin \frac{\pi}{2}=\frac{L i_{L}^{2}}{2}=-\omega \frac{C V_{C}^{* 2}}{2}=-\omega W_{C(\max )}
\end{array}\right\}
$$
where $W_{L(\max )}$ and $W_{C(\max )}-$ the maximum value of energy periodically accumulated in the magnetic field of the inductive machine and in the electric field of the capacitive machine.

On the basis of the law of conservation of energy, the reactive power of a combined machine is equal to the algebraic sum of the reactive powers of inductive and capacitive machines, i.e.

$$
P^{0}=P_{L}+P_{C}=\omega\left(W_{L(\max )}-W_{C(\max )}\right) .
$$

When $P^{0}=0$, there is a resonance of the voltages (for a series connection) and a current resonance (with a parallel connection).

In this expression for the resonance of the voltage and current have the form

$$
\begin{gathered}
V=\left[V_{R}^{2}+\left(V_{L}-V_{C}\right)^{2}\right]^{1 / 2}=i_{L}\left[R^{2}+(\omega L-\right. \\
\left.1 / \omega C)^{2}\right]^{1 / 2} \\
I=\left[I_{R}^{2}+\left(I_{L}-I_{C}\right)^{2}\right]^{1 / 2}=V_{C}^{*}\left[(1 / 2)^{2}+\right. \\
\left.(1 / \omega L-\omega C)^{2}\right]^{1 / 2}
\end{gathered}
$$

At resonance, the energy characteristics of the combined machine are the best, since the reactive power exchange between the inductive and capacitive subsystems takes place, and to create magnetic and electric fields the machine ceases to consume reactive power from external sources of energy.

If there are active electrotechnical materials of dual purpose - ferroelectromagnets, in the structure of the excitation system a combined inductive-capacitive electrical machine, then between the inductive and capacitive subsystems the electrophysical bond is nonlinear.

The Lagrange function $-\mathcal{L}_{L C}$ is not an additive function and can be expressed as: 


$$
\mathcal{L}_{L C}=\mathcal{L}_{L}+\mathcal{L}_{C}+\Delta \mathcal{L}_{L C},
$$

where $\Delta \mathcal{L}_{L C}$ a member of the Langrangian, takes into account the effect of one subsystem on another.

In our opinion, the necessary Lagrangian for combined inductive-capacitive EMF energy can be constructed by introducing a new interaction matrix (mutual influence) between the inductive and capacitive subsystems $-\widehat{K_{0}}\left(q, \Psi^{*}\right)$, which is expressed in the form

$$
\widehat{K_{0}}\left(q, \Psi^{*}\right)=\left|\begin{array}{llll}
K_{a m} & 0 & 0 & 0 \\
0 & K_{b n} & 0 & 0 \\
0 & 0 & K_{c h} & 0 \\
0 & 0 & 0 & K_{f p}
\end{array}\right|,
$$

where $K_{a m}=K_{0}\left(q_{a}, \psi_{m}^{*}\right), \quad K_{b n}=K_{0}\left(q_{b}, \psi_{n}^{*}\right), K_{c h}=$ $K_{0}\left(q_{c}, \psi_{h}^{*}\right)$ - express mutual influence between phase chains, $K_{f p}$ - determines the energy state of the combined inductive excitation system.

Since the active materials - ferroelectromagnets are completely installed in the rotor of a combined inductive capacitive EPM, in the general case $K_{f p}$ is a continuous functional of the thermal, electrophysical and mechanical characteristics of this material, expressed in the form

$$
K_{f p}=K_{f p}\left(\beta\left(i_{f}\right), \varepsilon\left(V_{p}\right), V_{o}, T\right),
$$

where $\beta\left(i_{f}\right)$ - magnetic permeability, $\varepsilon\left(V_{p}\right)-$ electrical permeability, $V_{0}$ - volume, $T$ - temperature of active material.

Basic analytical properties of matrix elements $\widehat{K}_{0}\left(q, \Psi^{*}\right)$ can be summarized as:

the elements $K_{a m}, K_{b n}$ и $K_{f p}$ are continuous periodic functions, have a continuous periodic derivative in the interval $0 \leq \gamma<\infty$ and satisfy the conditions

$$
\left.\begin{array}{c}
0 \leq\left|\mathrm{K}_{0}\left(\mathrm{q}_{\mathrm{i}}, \psi_{\mathrm{j}}^{*}\right)\right| \leq 1 \\
\lim _{\substack{\mathrm{q} \rightarrow 0 \\
\psi_{\mathrm{j}} \rightarrow 0}}\left|\mathrm{~K}_{0}\left(\mathrm{q}_{\mathrm{i}}, \psi_{\mathrm{j}}^{*}\right)\right|=00 \\
\int_{0}^{q_{\max }} \int_{0}^{*} T_{m} T_{m} d q_{i} d \psi_{j}^{*} \leq K^{0} \\
K^{0}>0 ; i=a, b, c ; j=m, n, h
\end{array}\right\}
$$

When the phase circuits are connected in series, the inductive subsystem as a voltage source is a "carrier system" with an active-capacitive load, and with a parallel connection, the "carrier" system is a capacitive subsystem as a current source with an actively inductive load:

$$
\left.\begin{array}{c}
\mathcal{L}_{L}=T_{L}-\Pi_{L} ; \mathcal{L}_{c}=T_{c}-\Pi_{c} \\
T_{L}=\frac{1}{2} i^{\tau} L i+i^{\tau} \widehat{K_{0}}\left(q, \Psi_{j}^{*}\right) V^{*} ; \Pi_{L}=\frac{1}{2} V_{\Re}^{*} C_{\Re} V_{\Re}^{*} ; \\
T_{c}=\frac{1}{2} V^{* \tau} C V^{*}+i^{\tau} \widehat{K_{0}}\left(q, \psi_{j}^{*}\right) V^{*} ; \Pi_{c}=\frac{1}{2} i_{\Omega}^{\tau} L_{\Omega} i_{\Omega}
\end{array}\right\}
$$

where $T_{L}, T_{c}$ - the effective electrokinetic energy of the inductive and capacitive subsystems, respectively;
$\Pi_{L}$ и $\Pi_{c}$ - energy of the electric field of the capacitive and magnetic fields of the inductive load; $i^{\tau} \widehat{K_{0}}\left(q, \psi_{j}^{*}\right) V^{*}$ - the volume energy between the two subsystems (the superscript $\tau$ denotes transposed matrices), $L$ - matrix of inductances, $i\left(i_{a}, i_{b}, i_{c}, i_{f}\right)-$ vector of currents of inductive machines, $C-$ capacitance matrix, $V_{\mathrm{g}}^{*}\left(V_{m}^{*}, V_{n}^{*}, V_{h}^{*}, V_{p}^{*}\right)$ - armature voltage armature vector, $L_{\mathrm{g}}$ - matrix of inductances, $i_{q}\left(i_{a}, i_{b}, i_{c}, 0\right)$ - vector of the armatures of the inductive machine:

$$
L_{\Omega}=\left[\begin{array}{cc}
L_{S S} & L_{S F} \\
0 & 0
\end{array}\right] ; \quad C_{\Omega}=\left[\begin{array}{cc}
C_{R R} & C_{R P} \\
0 & 0
\end{array}\right],
$$

On the basis of equations (1), (2), (14) - (18), the equations of electrodynamics and electromechanics for stationary modes of combined inductive capacitive EMF can be written in the following vector-matrix form: the equation of electrodynamics

$$
\left.\begin{array}{c}
\frac{d \Psi}{d t}+R_{L} i_{L}+K_{0}\left(q_{i}, \psi_{j}^{*}\right) \frac{d V_{C}^{*}}{d t}=V_{L C} \\
\frac{d Q^{*}}{d t}+G_{c} V_{c}^{*}+K_{0}\left(q_{i}, \psi_{j}^{*}\right) \frac{d i_{L}}{d t}=i_{C R}
\end{array}\right\}
$$

the equation of electromagnetic moments

$$
\begin{aligned}
M_{L C}=-\frac{P_{0}}{2}\left[i_{L} \frac{\partial L}{\partial \gamma} i_{L}^{\tau}+V_{c}^{*} \frac{\partial C}{\partial \gamma} V_{c}^{* \tau}\right. & \\
& \left.+i_{L} \frac{\partial K_{0}\left(q, \psi_{j}^{*}\right)}{\partial \gamma} V_{c}^{* \tau}\right]
\end{aligned}
$$

The simultaneous solution of the system of equations (20) and (21) completely determines the dynamic behavior of the generalized EMF model in any modes of interaction of their functional elements.

To study the combined EMF of a specific design, it is necessary to take into account all the structural features of their functional elements in the problems of computer-aided design in these equations. In this case, the dependencies of the elements of the interaction matrix on the physical characteristics and design parameters of the combined inductive capacitive EMF allow us to establish the use of the theory of experimental design [20].

\section{Conclusion}

1. The article presents differential controls of inductive capacitive electromechanical energy converters, on the basis of which, in a particular case, the equations of inductive and capacitive electric machines of alternating current are presented.

2. The use of achievements in inductive electric machines for the development of capacitive electric machines by means of inverse-coupled electrodynamics can significantly accelerate the development of the latter. The successful development of capacitive electric machines can become the basis for the creation of an inductive-capacitive machine with a working electromagnetic field. 
3. The article has established the possibility of optimizing the operating mode of an inductive generator with the help of a capacitive generator (or vice versa), with the formation of a parallel (or sequential) resonant circuit (the controlling parameter is the interaction matrix). A combined machine is a resonant machine and has the best energy performance.

A deeper application of the developed theoretical principles for generalized physico-mathematical, computer simulation, calculation of the optimal energy indices of combined inductively-capacitive EMF energy and their systems will be given in separate studies by the authors.

\section{References}

1 A.G. Iosifyan, Electricity, 12, 19-21 (1987); 9, 714 (1989)

2 I.P Kopylov, S.V. Gandilyan, Electrical Engineering, 9, 25-40 (1998)

3 I.P. Laptsevich, L.N. Grechikhin, N.G. Kuts, Energetika (Izv., Higher Educational Institutions of the CIS), 6, 64-77 (2012)

4 G.V. Nosov, S.V. Pustynnikov, Izvestiya Tomsk Polytechnic University, 4, 111-115 (2012)

5 V.A. Chelukhin, Izvestiya Tomsk Polytechnic University, 325 (4), 76-80 (2014)

6 A.A. Fraerman, UFN. 182 (12), 1345-1351 (2012)

7 N.V. Ter-Oganessin, Ferroelectrics. 438 (1), 101106 (2012)

8 B.J. Gallacher, J.S. Burdess, A.J. Harris, M.E. McNie, Proc. on Progress in Phys, 10-20 (2001)

9 P. Muralt, Rep. on Progress in Phys, 64, 13391388 (2001)

10 Nanotechnology in electronics (M.: The techno sphere, 2005)

11 V.E. Turkov, S.A. Zhukova, D.D. Riskin, Nanoand microsystem equipment. 12, 7-21 (2014)

12 J. Altman, Military Nanotechnologies: Possibilities for the Application and Preventive Control of Arms (M.: Technology, 2006)

13 A.N. Kovshov, Yu.F. Nazarov, I.M. Ibragimov, Fundamentals of nanotechnology in technology (M.: Academy, 2009)

14 H.S. Karayan, S.V. Gandilyan, Armenian J.Phys. 9 (3), 244-259 (2016)

15 G.S. Karayan, S.V. Gandilyan, Vestnik MPEI, 5, 63-70 (2017)

16 A.G. Iosifyan, G.L. Areshian, Dokl. AN Arm. SSR. 73 (1), 54-61 (1981)

17 S.V. Chelukhin, Izv. supreme. educational institutions (Electromechanics), 5, 48-52 (1987)

18 S.V. Gandilyan, Electricity. 8, 22-28 (1988)

19 S.V. Gandilyan, Izvestiya AN RF (Energy and Transport), 2, 50-62 (1993)

20 N.I. Sidnyaev, N.T. Vilisova, Introduction to the theory of experimental planning (M: Izd.-in MSTU him NE. Bauman, 2011) 